\title{
Coaching-Weiterbildung in Zeiten der Maske
}

\section{Eine Probebühne für einen selbstreflexiven, verlässlichen Umgang mit (beruflichen) Rollen, jenseits zeitgeistiger Authentizität}

\author{
Beate West-Leuer • Marga Löwer-Hirsch • Martin Gerstädt
}

Eingegangen: 11. Oktober 2020 / Angenommen: 2. November 2020 / Online publiziert: 22. Dezember 2020

(C) Der/die Autor(en) 2020

Zusammenfassung Coaching-Weiterbildungen in Zeiten der Ansteckung stehen vor neuen Herausforderungen. Besonders in großen Gruppen sind die gesundheitlichen Risiken allen Teilnehmer/innen sehr bewusst. Maskengebote durch die Veranstalter treffen jedoch nicht nur auf Zustimmung, sondern rufen auch Widerstand hervor. Besonders in Psychodynamischen Workshops mit Selbsterfahrungsanteilen war der Verzicht auf Vorgaben bisher Programm. Das Tragen der Maske beschneidet die mimische Kommunikation und erschwert Vertrauen. Doch die äußere Maske macht sichtbar, was jeder weiß: Ein Leben ohne Maskierung gibt es gar nicht. Wir alle inszenieren uns täglich in unterschiedlichsten Rollen. Diese Erkenntnis kann den Transfer erleichtern und den Anspruch an authentische Führung differenzieren.

Schlüsselwörter Corona-Krise - Große Gruppen · Mimische Kommunikation · Äußere Masken

\footnotetext{
Dr. phil. B. West-Leuer $(\bowtie)$

Wehler Dorfstraße 37, 41472 Neuss, Deutschland

E-Mail: west-leuer@t-online.de

Heinrich-Heine-Universität Düsseldorf, Düsseldorf, Deutschland

Dr. phil. M. Löwer-Hirsch

Simrockstr. 22, 40235 Düsseldorf, Deutschland

E-Mail: marga.loewer-hirsch@t-online.de

M. Gerstädt

Malmöer Straße 25a, 10439 Berlin, Deutschland

E-Mail: m-gerstaedt@t-online.de
} 


\section{Coaching training in times of the mask}

A rehearsal stage for a self-reflective, reliable handling of (professional) roles, beyond contemporary authenticity

Abstract Coaching training in times of contagion is facing new challenges. Especially in large groups, all participants are very aware of the health risks. Mask requirements by the organizers not only meet with approval, but also provoke resistance. Especially in psychodynamic workshops with parts of self-awareness, the waiver of specifications was previously the order of the day. Wearing the mask cuts off facial communication and makes trust more difficult. However, the outer mask reveals what everyone knows: There is no life without masking. We all stage ourselves in different roles every day. This knowledge can facilitate the transfer and differentiate the claim for authentic leadership.

Keywords Corona crisis $\cdot$ Large groups $\cdot$ Facial communication · Outer masks

\section{Prolog}

1. Seit der Mensch weiß, dass er kein Tier ist, trägt er Masken. Um Angst zu verbergen. Um zu erschrecken. Um sich stärker zu fühlen, als er ist. Um wild miteinander zu tanzen. Um anonymen Sex zu haben. Um Teil einer Gemeinschaft zu sein. Und meistens: um dem Tod zu trotzen. (Mayer 2020)

2. Aber dies Alles hätte anders sein können und die Griechen würden dennoch, wie ich zu behaupten wage, die Maske, die für uns eine so grosse Störung der Illusion ist, nicht abgelegt haben, denn dagegen erklärte sich bei ihnen ein eigenthümliches Schicklichkeitsgefühl. Wer bei ihnen nämlich eine fremde Persönlichkeit vorstellen oder auch nur die seinige verleugnen wollte, bedurfte dazu unter allen Umständen der Maske und dies nicht allein im Theater. (Geppert 1843, S. 260 f., zit. bei Dräger 2010, S. 50)

\section{Einführung}

Der Mensch ist das einzige Lebewesen, das mit einem Verstand ausgezeichnet ist, der es ihm ermöglicht, Krankheiten und deren Ansteckungsgefahren wissenschaftlich zu untersuchen und damit möglicherweise einzudämmen. Er ist aber auch ein Lebewesen, das sich wie kein anderes im Verlauf seiner Geschichte Lebensverhältnisse geschaffen hat, die ansteckenden Krankheiten enormen Vorschub leisten (vgl. Harari 2015). Während des Corona-Lockdowns haben wir unsere Weiterbildung teilweise ins Netz verlegt und „online“ durchgeführt. Damit haben wir, trotz anfänglichem Zögern und Zaudern aufgrund fehlender Übung mit der Technik, gute Erfahrungen gemacht (West-Leuer und Lewkowicz 2020). Als die der Pandemie geschuldeten Einschränkungen gelockert wurden, wurden allerdings auch die Rufe nach Präsenzveranstaltungen lauter. Für kleine Gruppen war dies kein Problem, konnten doch 
in den Räumen die Abstandsregeln eingehalten und die Masken am Platz abgelegt werden.

Ein Asset unserer Weiterbildung ist die Selbst-Erfahrung in großen Lerngruppen ab mindestens 16 Teilnehmenden. Bei dieser Gruppengröße wird es schwierig mit der Abstandsregel, obwohl der Raum $75 \mathrm{~m}^{2}$ Platz anzubieten hat. Nach der Ankündigung, dass unsere Weiterbildung nun wieder als Präsenzveranstaltung stattfinden würde, hatten wir schnell zwanzig Anmeldungen. Das ist untypisch für eine Weiterbildung mit psychodynamischer Ausrichtung, und als Veranstaltende mussten wir uns fragen, was zu tun sei.

\section{Ist die Große Gruppe noch zu retten?}

Die Überschrift ist doppeldeutig und müsste wie folgt lauten: Sind die Veranstaltenden noch bei Trost? Mit oder ohne Maske sind Gruppenveranstaltungen ein Ansteckungsrisiko, halten sich die Beteiligten doch acht Stunden am Tag in denselben Räumen auf. Und jetzt auch noch eine große Gruppe!

Durch die Zusammenarbeit von zwei Weiterbildungsinstituten können wir ein Modul als Großgruppe anbieten. In diesem Modul sind - neben Seminaren, Workshops und Übungen mit unterschiedlichen Lernformaten - Lerneinheiten der Großgruppenselbsterfahrung integriert. Das bedeutet die Konfrontation mit nicht immer angenehmen Großgruppenphänomenen. Aggressionen, Anonymisierungstendenzen, Spaltungs- und Hierarchisierungsmechanismen kennt jede und jeder aus organisationalen und gesellschaftlichen Kontexten. Dort werden sie in der Regel verdrängt, richten verdeckt gelegentlich umso größeren Schaden an. In den Lerneinheiten der Großgruppenselbsterfahrung sind die selektive Konfrontation mit diesen Phänomenen und ihre (teilweise) Bearbeitung zentral für den Lernprozess.

Nun sollte oder musste die Große Gruppe maskiert stattfinden. Tatsächlich ist die Maskierung eine unfreiwillige Konkretisierung eines zentralen Großgruppenthemas: Um mich vor den Anderen nicht angreifbar zu zeigen, verstecke ich mich hinter einer Maske, auch „Persona“ oder „soziale Rolle“ genannt (Goffman 1994).

Einige der Weiterbildungsinteressenten konnten und wollten in eine kleinere Parallelgruppe wechseln. Das war der sichere Weg. Andere wollten die Dynamik der Großen Gruppe am eigenen Leibe erleben, um latente Dynamiken in Organisationen und Unternehmen besser zu verstehen. Schließlich haben Organisationen in der Regel mehr als zwanzig Mitglieder. Die Bildung von Untergruppen, Spaltungsmechanismen in Gruppe-Gegengruppe-Denken, die Umwandlung von Angst in Aggression, insbesondere den Führungspersonen gegenüber, sind unvermeidlich. In den Organisationen werden diese Mechanismen mit Hilfe von Strukturen kanalisiert und bleiben so latent und beherrschbar. Werden Strukturen und Hierarchien - wie in New-Work-Ansätzen - als obsolet aufgegeben oder erst gar nicht eingeführt, sind selbstreflexive Erfahrungen umso hilfreicher, denn die stabilisierende Struktur entfällt. 


\section{So viel Reglementierung war nie! - Hygieneregeln als sicherheitsgebende Maßnahmen}

In unserer Weiterbildung wurden gleich zu Beginn der Veranstaltung vom Leitungsteam Hygienemaßnahmen ,erlassen“: Der Mundschutz wird im großen Seminarraum durchgängig getragen. Im Buffetraum dürfen sich maximal fünf Personen aufhalten, um sich dort zu bedienen. Zum Verzehr gilt es, sich in die kleinen Gruppenräume zu begeben. Bei der Kleingruppenarbeit darf nach Einnahme des Platzes die Maske abgenommen werden. Dies war in kleines Zugeständnis. Die Vorgaben würden den Teilnehmenden Sicherheit geben, so unsere Annahme und Intention. Wir mussten davon ausgehen, dass die uns unbekannten Gruppenmitglieder gegenüber Pandemie und offiziellen Hygieneregeln nicht einer Meinung waren. Pro und Contra in allen Schattierungen waren zu erwarten. Ohne strukturgebende Intervention der Weiterbildungsleitung besteht bei den Gruppenmitgliedern die unbewusste Sorge, dass sich Aggressionen frei flottierend gegen jede oder jeden richten, der beim Thema Maskenpflicht anderer Meinung ist als die Mehrheit.

\subsection{Interludium}

Das Wort „Maske“ stammt aus dem Arabischen: Mas-hara; der Narr, der Clown, der Possenreißer. Die Corona-Pandemie zwingt nun auch die Politik, von Maskenverbot zum Maskengebot zu wechseln, und sie nennt dies einen Kulturbruch. Dieser dürfte darin bestehen, dass künftig nicht mehr der Maskierte der Außenseiter, der Künstler, der Horrorclown oder Demonstrant ist, sondern im Gegenteil derjenige, der die Maske verweigert, sich und die anderen nicht schützt, als asozial gelten wird (Mayer 2020).

Durch die Verordnung der Maskenpflicht wird die Richtung der Aggressionen in der Weiterbildungsgruppe auf die Führung gelenkt. Dies vermittelt Sicherheit, ist aber nicht angenehm für die Seminarleitung. Enttäuschung und auch Ärger werden in den informellen Untergruppen an- und ausgesprochen. Die Leitung spürt eine mehr oder weniger ablehnende Atmosphäre (De Maré 1977).

In psychodynamischen Weiterbildungskonzepten sind solch strikte Vorgaben nicht üblich. Selbst- und Fremd-Erleben benötigen einen „offenen“ oder ,potentiellen“ Raum (Winnicott 1988 [1965]). Schon eine Aufforderung zum Feedback kommt einer Verzerrung gleich. Denn die Gruppenmitglieder ,maskieren“ ihre unfreiwilligen Feedback-Äußerungen, bewusst und unbewusst. Die äußere Realität wird in der psychodynamischen Gruppenselbsterfahrung daher nur durch das Setting repräsentiert. Trotz der Geringfügigkeit des gesetzten Arrangements (Zeitvorgaben, Anordnung der Stühle) beeinflusst es die Kommunikation und das Übertragungsund Gegenübertragungsgeschehen zwischen Leitung und Gruppe.

Im vorliegenden Fall kommt für das Großgruppenlabor durch die Maskenpflicht eine weitere, nicht in Frage zu stellende, äußere Regel hinzu. Sie gilt für die Gruppenmitglieder, aber auch für die Leiterin des Labors, die ihre Arbeitsweise anpassen oder auch umstellen muss. 


\section{Fallbeispiel ,Großgruppenlabor“}

Die Gruppenmitglieder kennen sich untereinander nicht oder nur online. Um zu einer Arbeits- bzw. Lerngruppe zusammenzuwachsen, bedarf es eines gewissen Maßes an ,gesundem Vertrauen“, das heißt weder blindes Vertrauen, noch unbegründetes Misstrauen. Ein solch balancierendes Vertrauen entsteht als Ergebnis zwischenmenschlicher - auch mimischer - Interaktionen, die beides enthalten: Empathie und Konfrontation. Erst wenn geklärt ist, dass sich jede und jeder so sehr blamieren kann, wie sie mag, kann das verbindende Lernziel, die Qualifikation eines psychodynamischen Coachs zu erwerben, gemeinsam in Angriff genommen werden.

„Nun sitzen wir hier in einer Situation, in der uns die Vernunft sagt, dass Maskentragen sinnvoll ist, um eine Ansteckungsgefahr zu verringern; gleichzeitig sträuben sich Gefühle dagegen, derart aus der Bahn der gewohnten Kommunikationsformen in der körperlichen Begegnung von Mensch zu Mensch und in größeren Gruppen von Menschen geworfen zu werden." So oder ähnlich leitete die Leiterin des Labors das Teamtraining-Live ein und drückte selbst ihr Befremden darüber aus, mit Maske zu arbeiten. Diese einleitenden Worte zur Maskenpflicht wurden jedoch dynamisch untereinander und in Bezug auf Leitung kaum aufgegriffen. Es war so, als habe man einen Stein ins Wasser geworfen, der keinerlei Wellen hinterlässt. Auf einer Tiefenebene wurden aber Erfahrungen reaktiviert, der denen eines Kleinkindes gleichkommen, dem gesagt wird: Fass nicht in eine Steckdose. Das Kind muss vertrauen oder vertraut auch nicht; denn es weiß nicht, warum es das nicht darf, warum es nicht hineinfassen darf.

Zunächst geht der Prozess der Kontaktaufnahme über den Austausch von „Nettigkeiten" kaum hinaus, scheint zu stagnieren. Erst als ein Teilnehmer sich traut, zu sagen, er fühle sich sehr unangenehm an Abhängigkeitsgefühle gegenüber den Elternfiguren aus seiner Kindheit erinnert und diese Gefühle seien alles andere als angenehm, konnte sich langsam an die Leitungsvorgaben herangetastet werden. Ein erneutes Ansprechen der Maskenpflicht durch die Leiterin des Labors ließ nun eine Bearbeitung in Gang kommen, die essentiell für die Bildung eines Gruppengefühls wurde. Möglich wurde es vor allem durch die Thematisierung der Vertrauensfrage in Leitung.

Einige Gruppenmitglieder trauen sich nun, ihr Erleben und ihre Reaktionen zu benennen. Sie beklagen sich über die Erschwernis, mit den Anderen in einen lebendigen Kontakt zu kommen, bei allem Verständnis für das Dilemma der Verantwortlichen. Und sie berichten, dass sie nur ein „Gefühl“ für diejenigen Gruppenmitglieder entwickeln konnten, mit denen sie unmaskiert in einer Untergruppe zusammengearbeitet haben. Sie äußern Enttäuschung und auch Ärger über das Maskengebot; schließlich sei von den Veranstaltern „versprochen“ wurde, dass der Raum groß genug sei; vielleicht fühlen sie sich ein wenig hintergangen.

Auf Nachfragen der Leiterin des Labors wird deutlich, dass das Team der Veranstalter aus den Arbeitsprozessen in den Kleingruppen teilweise und bewusst ausgeschlossen wird. Dort sei man unmaskiert und bleibe unter sich, was auf die Besetzung neuer, von Leitung unabhängiger „Spiel- oder Freiräume“ hindeutet. Konflikte mit der Weiterbildungsleitung können konstruktiv bearbeitet werden; Teilnehmerinnen sprechen z. B. an, dass sie sich im Vorfeld vom Veranstalter nicht ,gut genug“ - auch 
im Sinne Winnicotts (1988 [1965]) - informiert fühlen. Es können subjektive ÄuBerungen und Wahrnehmungen Einzelner diskutiert werden, die aus persönlichen Beziehungserfahrungen stammen und auf die Leitung übertragen wurden.

Für die Weiterbildungsleiterin bleibt am Ende auch ein Gefühl von Verlust und Trauer, als sei die Weiterbildung wesentlicher Erkenntnispfade beraubt. Die Evaluation des Moduls zeigt jedoch, dass die Gruppenselbsterfahrung im Labor für die Gruppenmitglieder mehrheitlich ein ausnehmend positive war und zur Bildung eines kohärenten Gruppengefühls beigetragen hat. Warum denn dann der Widerstand gegen das Tragen der Maske?

\section{Wir alle spielen Theater - auch in der Coaching-Weiterbildung}

Die Aussagen der Gruppenmitglieder lassen erkennen, dass die eigene Maske zwar eine Unbequemlichkeit darstellt; als eigentliche Störung wird die Maskierung der Anderen empfunden. Müssen wir die Mimik unserer Interaktionspartner also sehen, um Vertrauen zu entwickeln? Oder ist die Maske vielleicht sogar eine hervorragende alternative Erfahrungsmöglichkeit, ein Geschenk geradezu? Die gängige Behauptung einer ,ganzheitlichen“ Sicht auf zwischenmenschliche Interaktion, die das Fehlen der vielen tausend Gesichtsreaktionen beklagt, leistet einer Fiktion Vorschub. Die Corona-bedingte Maske macht vielmehr äußerlich sichtbar, was wir bereits wissen: Ein Leben ohne „Maskierung“ gibt es nicht.

\subsection{Persona und Schatten}

Im Kommunikationsverständnis des Symbolischen Interaktionismus (Mead 1993 [1934]; vgl. Goffman 1994) findet Zwischenmenschlichkeit immer als Inszenierung statt: Wir alle spielen Theater. Es wird kein Satz geäußert, ohne dass Wortschatz und Tonfall dem angepasst werden, was wir aus der Rollenidentität der Zuhörer schließen, ,und zwar unbesorgt darüber, dass es sich dabei um Vermutungen handelt“" (Goffman 1994, S. 62f.).

Aus psychodynamischer Sicht sind es frühe Erfahrungen der Scham, die zu einer Aufspaltung des Selbst in Rollen führen. Diese Aufspaltung ist ein Muster, das sich in der Kindheit herausbildet, das aber das ganze Erwachsenendasein in allen Gemeinschafts- und Normzusammenhängen durchzieht. Wir spalten uns auf in ein öffentliches und ein privates Ich, in Persona und Schatten. Persona ist, was wir nach außen vorgeben und was uns in der Gemeinschaft Wert verleiht. Schatten ist, was wir hinter dieser Fassade verborgen halten, weil es uns - so die unbewusste Annahme - unwert machen würde, eben das, was in der Scham so peinvoll enthüllt wird (Jung 1950, vgl. West-Leuer 2010). Da wir mit Notwendigkeit beides zugleich sind, Persona und Schatten, können wir diese Aufspaltung als eine Grundverfassung unseres Daseins, als ein Existenzial verstehen (Schüttauf 2008, S. 852 f.).

Norbert Elias (1976) hat gezeigt, wie das Individuum, im Prozess der Zivilisation, in Haltung und Bewegung einer Formung unterworfen wurde, um seine Triebund Affektkontrolle zu erhöhen. Kontrolliert wird aber nicht nur, was Anderen von mir gezeigt werden soll oder nicht, sondern auch vor mir selber finden Versteck- 
spiele statt, d.h. mein Körper kann etwas ausdrücken, was mir selbst nicht bewusst ist und nur durch Spiegelung von außen bewusst werden kann. Mimik, Haltung, Bewegungen zeigen etwas, das dem Bewusstsein nicht freigegeben ist. Körper und Körperlichkeit lassen sich nur teilweise ,,verstecken“, schon kaum unsere Regungen und der Ausdruck unserer Gefühle. Der Körper drückt manchmal mehr aus, als uns lieb ist (vgl. Löwer-Hirsch 2012).

\subsection{Einschränkungen in der Selbstinszenierung und Auswirkungen auf das Regressionsniveau der Gruppendynamik}

So verstanden ist der Widerstand gegen das Tragen der Maske bedingt durch den Verlust der Möglichkeit, sich als „Persona“ situationsangepasst und vorgeblich ,,authentisch“ mimisch zu inszenieren, begleitet von der Illusion, den eigenen Schatten zu verbergen. Gleichzeitig ist der Widerstand bedingt durch den Verlust der Möglichkeit, den Anderen an seiner Mimik bis in den hintersten Winkel seiner Seele durchschauen und sich entsprechend schützen zu können, - wenn diese Möglichkeit auch nur eine sozial weitverbreitete Illusion darstellt. Denn nicht erst seit Stephen Kings Horrorgeschichte (King 2011 [1986]) verbirgt sich das ES als böses Anderes hinter der Maske des bunten Clowns und ist doch auch das uns eigene Unheimliche (vgl. Freud 1919). Und das macht Angst.

Dass diese Verluste den Aufbau von Vertrauen in der Gruppe erschweren, zeigt sich in der Gruppendynamik des Fallbeispiels am Regressionsniveau. Um dies zu zeigen, werden die fünf Tiefenebenen nach Foulkes (1986 [1964]; vgl. Lemche 1993) herangezogen: Auf der Aktualebene, die Forumcharakter hat und aus den gegenwärtigen Erfahrungen und der zwischenmenschlichen Gefühlsdynamik der Teilnehmenden besteht, herrscht der Austausch von Nettigkeit vor, um dann direkt auf die Übertragungsebene kindlicher Erfahrungen mit Elternfiguren zu regredieren. Die dritte Tiefenebene oder Projektionsebene ist typisch für große Gruppen. Hier inszenieren sich Spaltungsphänomene zwischen den Untergruppen. Doch im Fallbeispiel einigt sich die Gruppe schnell und äußert Misstrauen gegenüber der Leitung. Gefühlsregungen wie Angst oder Aggression richten sich entsprechend auch nicht gegen Untergruppen, sondern direkt gegen die Leitung.

Während das Geschehen auf der Aktualebene weitgehend bewusst und das auf der Übertragungsebene vorbewusst, aber dem Bewusstsein zugänglich ist, ist die Projektionsebene im Wesentlichen unbewusst, gelegentlich durch die Interpretation der begleitenden Gefühlsregungen ansprechbar. Die vierte Tiefenebene oder Körperebene verrät dagegen mehr, als der Person lieb ist (s. oben). Als die Leiterin des Labors eine Teilnehmerin, die vorgibt, völlig entspannt zu sein, mit den folgenden Worten auf ihre ,kickende“ Fußbewegung hinweist: „Vielleicht hoffe sie insgeheim, dass etwas mehr ,Aktion" von der Leitung ausgehe", fühlt sich diese wie ertappt. Die Primordialebene oder fünfte Ebene wurde nicht angesprochen. Hier finden sich Urformen und Urbilder, so genannte „Imagines“, die das kollektive Unbewusste der sozialen Gruppe als ererbte Grundlage jeder Persönlichkeitsstruktur bilden. Hier liegt der unaussprechliche Horror, dem maskierten Anderen und dem maskierten Selbst zutiefst verborgen, und steuert die anfängliche Angst vor der Maskierung, bis sie durch „Rationalisierung“ handhabbar wird. 
Zusammenfassend war der Wegfall der Aktualebene besonders regressionsfördernd. Die Fokussierung auf die Leitung als Projektionsfläche ist die unmittelbare Folge und in der Ausschließlichkeit auffällig. Das Geschehen auf der Übertragungsebene wirkte stabilisierend, sodass ein tieferes Sich-auf-den Prozess-Einlassen möglich wurde. Die Körperebene ist in jeder Klein- wie Großgruppe zu beobachten und - und wenn angemessen adressiert - hilfreich für den Prozess. Der Einfluss der Primordialebene wird durch fiktive Analogiebildung wahrscheinlich (vgl. Prolog 1).

\subsection{Die Corona-bedingte Maskierung als Gewinn? Ein Versuch}

In der Großgruppe besteht die Wirkung der Maskierung auch darin, dass sie den Träger der Maske seiner mimischen Individualität beraubt und ihn als Inhaber einer Rolle, die er in der Gruppe einnimmt, sichtbar macht. Seine Rolle ist der Text, den er spricht. Der Text, also der Sprechakt, ist die Verbindung zwischen der Person des Sprechers und dem Unbewussten der Gruppe. So kann die Corona-bedingte Maske - weg vom Horror und hin zur Bearbeitung (vgl. Prolog 2) - eine Funktion übernehmen, die an die Maske im antiken griechischen Theater erinnert: In dem, was der Sprecher sagt, spricht er nicht für sich, sondern für die Gruppe und zeigt dies an durch das Verhüllen der eigenen Person, Persona wie auch Schatten.

In Anlehnung an das Abstinenzgebot des psychoanalytischen Settings, in dem der Analytiker weder Persona noch Schatten ausagiert, lädt die Maske ein zu einem auf den Sprechakt fokussierten Zugang zum Unbewussten in der jeweiligen Szene und dient der Komplexitätsreduzierung. Der Gewinn des Corona-bedingten, unfreiwilligen Experiments „Maske“ könnte nun darin liegen, dass die Gruppe zwar schnell regrediert, aber, weil der Sprechakt in den Vordergrund der Interaktion tritt, dieses regressive Abtauchen verbalisiert und durch das Verbalisieren die Regression auch schnell wieder auflöst. Mit der verordneten Maske in der Weiterbildung zu leben und diese als Zugang zum kollektiven Unbewussten zu nutzen, ist vor diesem Hintergrund ein Hinweis auf seelische Reife im Umgang mit Rolle, Persona und Schatten.

\section{Transfer in die Beratungstätigkeit}

Bei Coaching-Anfragen haben wir es vordergründig weniger mit dem privaten Schatten als mit der öffentlichen Persona der Klienten zu tun. Dabei ist die Analyse, wie berufliche Rolle, persönliches Ich und Schatten bis in die Leiblichkeit hinein interagieren, immer schon ein Herzstück psychodynamischer Weiterbildung und Beratung (vgl. Löwer-Hirsch und West-Leuer 2017; West-Leuer 2003; Löwer-Hirsch 2003).

In der Reflexion konnte das Tragen der Corona-bedingten Maske bei den Teilnehmer/innen der Weiterbildung einen bewussteren Umgang mit den eigenen Inszenierungen in beruflichen und privaten Rollen anstoßen und Funktionalität und Radius dieser Inszenierungen erweitern. Die Sicht auf die maskierten Gruppenmitglieder als Rollen- und Funktionsträger im Auftrag der Gruppe machen einen Transfer auf die berufliche Situation von Klienten leicht nachvollziehbar. Entsprechend werden 
Rolleninszenierungen der Klienten nicht unbedingt als Makel, sondern als Professionalisierungsmerkmal von Führung angesehen; sie können im Coaching thematisiert und - da wo sich Dysfunktionales aus Persona oder Schatten eingeschlichen hat durchgearbeitet und angepasst werden. Denn die zeitgeistige Authentizitätsgläubigkeit mimischer oder ,ganzheitlicher“ Inszenierung ist auch eine Maske, allerdings eine, die Echtheit behauptet. In der Begegnung der Rolleninhaber „Coach und Klient“ oder „Führungskraft und Mitarbeitende“ erschwert oder vermeidet diese Echtheitsbehauptung den Zugang zum Unbewussten.

Funding Open Access funding enabled and organized by Projekt DEAL.

Open Access Dieser Artikel wird unter der Creative Commons Namensnennung 4.0 International Lizenz veröffentlicht, welche die Nutzung, Vervielfältigung, Bearbeitung, Verbreitung und Wiedergabe in jeglichem Medium und Format erlaubt, sofern Sie den/die ursprünglichen Autor(en) und die Quelle ordnungsgemäß nennen, einen Link zur Creative Commons Lizenz beifügen und angeben, ob Änderungen vorgenommen wurden.

Die in diesem Artikel enthaltenen Bilder und sonstiges Drittmaterial unterliegen ebenfalls der genannten Creative Commons Lizenz, sofern sich aus der Abbildungslegende nichts anderes ergibt. Sofern das betreffende Material nicht unter der genannten Creative Commons Lizenz steht und die betreffende Handlung nicht nach gesetzlichen Vorschriften erlaubt ist, ist für die oben aufgeführten Weiterverwendungen des Materials die Einwilligung des jeweiligen Rechteinhabers einzuholen.

Weitere Details zur Lizenz entnehmen Sie bitte der Lizenzinformation auf http://creativecommons.org/ licenses/by/4.0/deed.de.

\section{Literatur}

De Maré, P. (1977). Die Politik großer Gruppen. In L. Kreeger (Hrsg.), Die Großgruppe (S. 140-153). Stuttgart: Klett-Cotta.

Dräger, C. (2010). Vom Maskenkult zur Theatermaske. Haben die antiken Theatermasken die Stimme verstärkt? Inauguraldissertation. zur Erlangung der Würde des Doktors der Philosophie der Universität Trier. www.DraegerCorinna20100212.pdf. Zugegriffen: 28. Sept. 2020.

Elias, N. (1976). Über den Prozess der Zivilisation. Frankfurt/M.: Suhrkamp.

Foulkes, S. H. (1986). Gruppenanalytische Psychotherapie. Frankfurt/M.: Fischer.

Freud, S. (1919). Das Unheimliche. Psychologische Schriften. StA IV. (S. 241-274). Frankfurt/M.: Fischer. Goffman, E. (1994). Interaktion und Geschlecht. Frankfurt/M.: Campus.

Harari, Y.N. (2015). Eine kurze Geschichte der Menschheit. München: Pantheon.

Jung, C. G. (1950). Aion. Beiträge zur Symbolik des Selbst. GW, Bd. 9/2. Olten, Freiburg/Br: Walter.

King, S. (2011). Es. München: Heyne.

Lemche, E. (1993). Der gestalttheoretische Aspekt und sein Einfluß auf die Interventionsweise bei S. H. Foulkes. Gruppenpsychotherapie und Gruppendynamik, 29, 70-102.

Löwer-Hirsch, M. (2003). Das Unbewusste in Organisationen und der intersubjektive Ansatz. In B. WestLeuer \& C. Sies (Hrsg.), Coaching - Ein Kursbuch für die Psychodynamische Beratung (S. 25-43). Stuttgart: Klett-Cotta.

Löwer-Hirsch, M. (2012). Körperlichkeit und Supervision. Die Arbeit mit Menschen aus „Fleisch und Blut“ - ,versehrt“" und „,unversehrt“. In W. Weigand (Hrsg.), Philosophie und Handwerk der Supervision (S. 123-136). Gießen: Psychosozial.

Löwer-Hirsch, M., \& West-Leuer, B. (2017). Psychodynamisches Coaching für Führungskräfte. Einzelund Gruppencoachings in Theorie und Praxis. Wiesbaden: Springer.

Mayer, W. (2020). Vermummungsgebot. Focus magazin Nr. 16, 20.4.2020. https://www.focus.de/kultur/ leben/corona-vermummungsgebot_id_11872567.html. Zugegriffen: 28. Sept. 2020.

Mead, G.H. (1993). Geist, Identität und Gesellschaft. Aus der Sicht des Sozialbehaviorismus. Frankfurt/M.: Suhrkamp.

Schüttauf, K. (2008). Die zwei Gesichter der Scham. Psyche, 62, 840-865. 
West-Leuer, B. (2003). „Tief statt breit“: Coaching anders als die anderen. In B. West-Leuer \& C. Sies (Hrsg.), Coaching - Ein Kursbuch für die Psychodynamische Beratung (S. 148-170). Stuttgart: KlettCotta.

West-Leuer, B. (2010). Was ist einer? Was einer nicht? - Hysterie und Narzissmus im Film und auf der Bühne. In K. Tiedemann \& F. M. Raddatz (Hrsg.), Reality Strikes Back II. Tod der Repräsentation (S. 110-127). Köthen: Theater der Zeit.

West-Leuer, B., \& Lewkowicz, E.-M. (2020). „Einfach mal probieren“. Ein persönlicher Erfahrungsbericht über Online-Coaching aus der Werkstatt Psychodynamischer Beratung. https://www.coachingmagazin.de/beruf-coach/erfahrungsbericht-online-coaching. Zugegriffen: 4. Sept. 2020

Winnicott, D. W. (1988). Reifungsprozesse und fördernde Umwelt. Frankfurt/M.: Fischer.

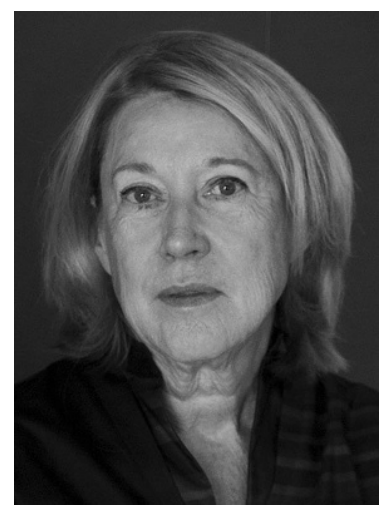

Dr. phil. Beate West-Leuer Psychologische Psychotherapeutin, Senior Coach (DBVC, IOBC), Supervisorin (DGSv), Leiterin des Instituts Psychodynamische Organisationsentwicklung + Personalmanagement Düsseldorf e. V. (POP), Stellv. Vorsitzende der Akademie für Psychoanalyse und Psychosomatik Düsseldorf, Lehrbeauftragte der HeinrichHeine-Universität.

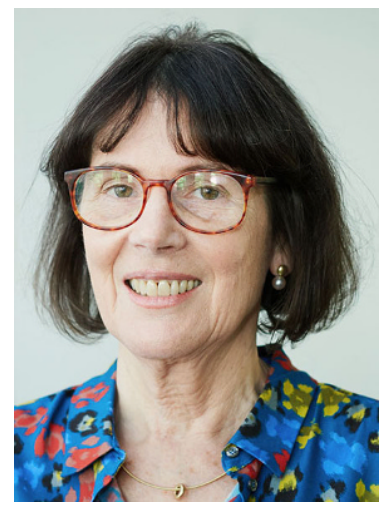

Dr. phil. Marga Löwer-Hirsch Psychologische Psychotherapeutin, Supervisorin (DGSv), Senior Coach (DBVC), Gründerin und Vorstandsmitglied des Instituts für Analytische Supervision an der Akademie für Psychoanalyse Düsseldorf, Lehrbeauftragte an der Internationalen Psychoanalytischen Universität Berlin. 


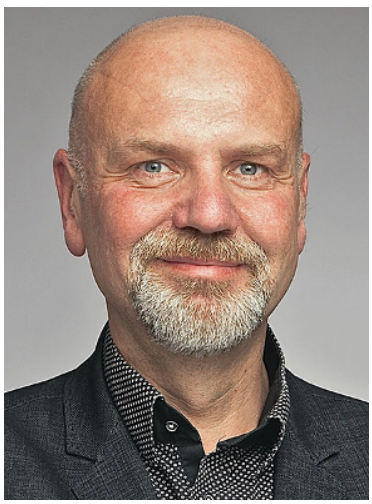

Martin Gerstädt Diplom-Sozialpädagoge, Supervisor, Coach, Organisationsberater. 